\title{
AUTOMATED VISUAL FAULT INSPECTION OF OPTICAL ELEMENTS USING MACHINE VISION TECHNOLOGIES
}

\author{
Hong-Dar Lin ${ }^{1 *}$, Hsing-Lun Chen ${ }^{1}$ \\ ${ }^{1}$ Department of Industrial Engineering and Management, Chaoyang University of Technology, Taiwan
}

Light-emitting diode (LED) lenses are one kind of common optical elements applied in many modern electronic devices. The LED lens with textured and uneven surface is hard to inspect appearance faults. This research suggests a wavelet packet transform-based partial least squares method to inspect visual faults of optical lenses with textured and uneven surfaces. Three major procedures are conducted to complete the process of fault detection. Firstly, a testing image is transformed to wavelet pack domain and the wavelet characteristics of the sub-band images are extracted. Secondly, the partial least squares scheme is used to multivariate transform with wavelet characteristics to obtain latent images. Thirdly, the latent images are fitted by a regression model to produce a predicted image. After comparing with the original image, we can obtain the residual image where the appearance faults have been separated. Thus, the intricate faults embedded in the complicated appearances of optical lenses could be precisely identified by the suggested method. The effectiveness and accuracy of the developed method are confirmed by expert assessments, as well as by comparative analysis with the known methods in the field of spatial localizations and classification effects of fault inspection.

Key words: Automated fault inspection; Optical elements; Visual fault; Machine vision; Imaging; Inspection; Automation; Faults; Lenses; Texturing; Wavelets

\section{INTRODUCTION}

Optical elements are employed to change the condition of light by a diversity of ways comprisingreflecting, focusing, filtering, etc. Lenses areopticalelements with ideal or roughaxial symmetryemitting and refractinglight, which converges or diverges the ray. Optical lenses withcrystal clearappearances areconstructed from optical materials and are bent for converging or diverging emitted beams from an object. Lenses are representatively composed of glass or clearplastic. The intention of freeform optical appearance has been abroadutilized for the glass or plastic optical elements in widespread lighting systems. Optical lensesareextensively applied inmobile phones, computers, vehicles, cameras, light-emitting diodes (LEDs), etc. LED is a semiconductorapparatus that launches visual ray when an electric flowtransits the semiconductor chip. The creation of LED has guided the considerablebenefit and provided the enormousanswerwith regard to both energy reduction and environmental conservationfrom now to later. Representativeutilizations of LED elements comprise indicator lamps, LCDscreen backlighting, automotive signal lighting devices, etc. The effects of LED lensescontainaccurate control over the rays of light, aesthetic appearance, and preventing the waste of light and light contamination. An LED can transmit light to far distance due to the support of lens focus function. Thence, LED lenses are created to enhance the light scattering issues of LEDs and they are abroadused to vehicle lights, flashlights, traffic lights, etc. Appearance differences among the clear lenses, diffused lenses, and textured lenses of LEDs are various designs for providing the lighting energy allocation in the regions of interest and decreasing the unbearable glare influence for the ordinarylightingsystems. Figure 1 shows LED lenses and a LED product with textured and uneven surfaces. Visual faults on the uneven surfaces are difficult to be inspected out for professional censors due to repetitive texture patterns on lens surfaces. The LED products are being manufactured in more compact sizes, which cause more trouble of inspecting products. The most common inspection method for appearance
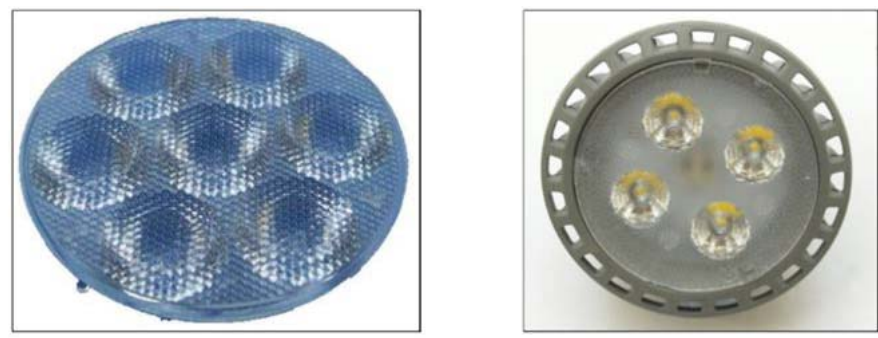

Figure 1: LED lenses and LED product with textured and uneven surfaces.
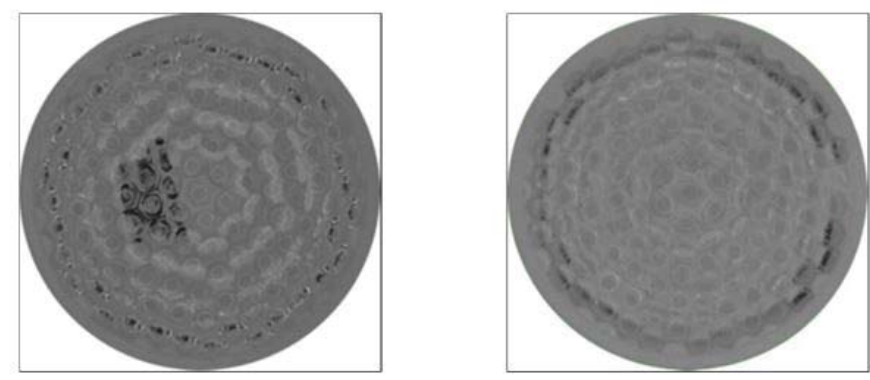

Figure 2: A defective LED lens image and a defect-free image with appearance faults. 
faults on LED lenses is manual visual inspection. Lens inspection needs particular physical circumstances, especiallywith regard to lighting. In the actualoperating conditions, each censoredlens is guided into the censor's sight area. The lenses are circular and textured andthe visual faults to be examined could be situated on the outside surfacesof the lenses or interior. The textured lens has the appearance of repeated small patterns in the captured image which is more complicated than those of the clear lens and diffused lens. It is more hard to correctly inspectout visual faultsinlaid in the complex repetitive patterns. The mostof faultsare not only quite small but also they are highlyvarious and can assume varying shapes. Figure 2 shows a defective LED lens image and a defect-free image with appearance faults. Manual inspection task is boring, time-consuming and extremelyrelying on the censors' skills. Wrong judgments are likely conducteddue tocensors' subjectivity andeye tiredness. Since the LED lens has the appearance of repeated small circles, those apparent textures make the fault detection task harder when visual faults are embedded on the uneven surfaces of structural textures. Therefore, this study suggests a wavelet packet transform-based partial least squares scheme to conquer the problems of usual computer vision systems.

The remainder of the paper is organized as follows: First, we go over the literature on optical methods of image processing for fault detection. Second, we explain the suggested image models for inspecting faults on LED lenses. Third, we carry out the experiments and assess the performance of the suggested schemes with known methods. Four, we present the conclusion and the future work.

\section{LITERATURE REVIEW}

Inspection of appearance faults has turned intoan essential work for producers who try hard to enhance product grade and manufacture efficiency [1-2]. Fault detection techniques ordinarily classified into two main categories: the spatial domain methods and the frequency domain methods. For the spatial domain technologies, Lin and Chiu [3] proposed anautomated vision system to search mass centers of chips, detect cutting lines and predict process regulation plans for the high-speed dicing of electronic passive components. Lin and Hsieh [4] developed a new vision system based on slight deviation control techniques to detect distortion defects on curved mirrors of vehicles.

Regarding technologies in the frequency domain, Chiu and Lin [5] introduced a visual inspection system with image models based on wavelet transformation filtering for automatic detection of area flaws on surfaces of capacitive touch screens. Li and Tsai [6] implemented a wavelet-based discriminant measure todetect defects in multi-crystalline solar wafer images with inhomogeneous texture. Lin and Ho [7] suggested an original method that employs discrete cosine transform-based enhancement for the inspection of pinhole faults on passive component chips.

Some studies investigated the surface fault inspection of LED related products. Lin and Chiu [8] developed a computer-aided inspection system that usesblock discrete cosine transform and grey relational analysis for the precisedetection of tiny flaws arose in the domed exteriors of LED epoxy-packing. Chiu and Lin [9] applied block discrete cosine transform, Hotelling's T-squared statistic, and grey clustering techniqueto inspect surface flaws on LEDs with clear lenses. Most of the current studies pay attention to inspections of LED lenses with clear surfaces, mirrors, andtransparent glass. These studies do not inspect flaws with the characteristics of small faults on textured LED lenses with uneven surfaces. Accordingly, we suggest a new method combining wavelet packet transform and partial least squares techniques for fault inspection on the textured and uneven surfaces of LED lenses.

The wavelet transform allows a time-frequency decomposition of the input signal, but the degree of frequency resolution in the wavelet transform is representatively regarded too rough for empirical time-frequency analysis [10]. The wavelet packet transform provides a computationally efficient option with sufficient frequency resolution. In the wavelet packet transform, the filtering functions are also implemented to the detailed components. Thus, the wavelet packets offer a sub-band filtering of the input signal into increasingly detailed equal-width intervals [11]. Kim and Kang [12] used wavelet packet concept and Gaussian mixture structure to perform texture classification and segmentation.

Partial least squares method was initially invented by Wold [13]. This method is a multivariable statistical technique carrying some connection to principal components analysis and it searches a linear regression model by transforming the predicator variables and the response variables to a new domain [14]. Because both the predicator and response data are transformed to new domains, the partial least squares relatedtechniques are familiar as bilinear factor models. The partial least squares method is employed to decrease the dimensionality of data, to discover the latent variables by catching the greatest variance in the data and accomplishes the maximum correlation between the predicated variables and observable variables [15]. Li et al. [16] employed the partial least squares method to inspect and grade surface defects of fruits in computer vision fields. This method has been successfully applied in diverse fields including process modeling, fault detection, process monitoring and it deals with noisy and highly correlated data.

\section{MATERIALS AND METHODS}

This study suggests a wavelet packet transform-based partial least squares scheme to inspect visual faults on uneven surfaces of LED lenses. The paper extends the earlier work [17] of the authors by a considerable amount 
of discussion and further experiments. A testing image is transformed to wavelet pack domain and the wavelet characteristics of the sub-band images are extracted.Then, the partial least squares model is applied to multivariate transform with wavelets features to obtain latent images. Finally, the latent images are fitted by a regression model to produce a predicted image. And the predicted image compares with the original one to obtain the residual image where the visual faults have been separated. Thus, the visual faultsembedded in the complicated appearances of optical lenses can be correctly identified by the suggested method.

\section{Image Modeling \& Analysis}

The inspection work of this study is to detectvisualfaults on uneven surfaces of optical lenses. Most of these unexpected faults are very small in size and cannot be depicted by accurateestimates, thus making computer-aided fault detection hard. With good time-frequency differentiation capability and flexible time-frequency windows, wavelet transforms are now diffusely adopted for analysis of various signals in time and frequency domain concurrently [11]. In the wavelet decomposition process, the usual stage decomposes the approximated components into two portions. After the dividing, we acquire a vector of approximated components and a vector of detailed components and both of the vectors are at a rougherestimate. The message missed between two consecutive approximations is caught in the detailed components. The next stage includes dividing the new approximated component vector; consecutive details are never re-examined. In the relevant wavelet packet circumstance, each detailed component vector is also divided into two portions using the identical procedure as in approximated vector dividing. Figure 3 shows a testing image taken wavelet transform and wavelet pack transform both with decomposition level 2. It indicates that the wavelet pack transform has the advantages of being fine enough to abstractessentialmessage from the decomposed components and representing generalization of multi-resolution decomposition. Due to decomposition of only the approximated components at each level, the results of frequency resolution in higher level wavelet decompositions (e.g. A1 and D1) are less advisable in

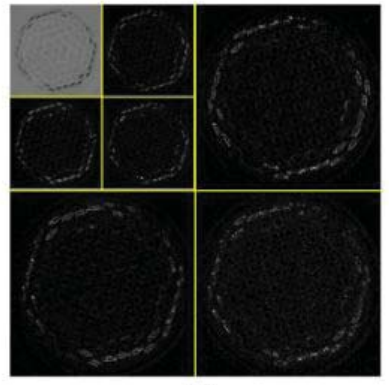

(a)

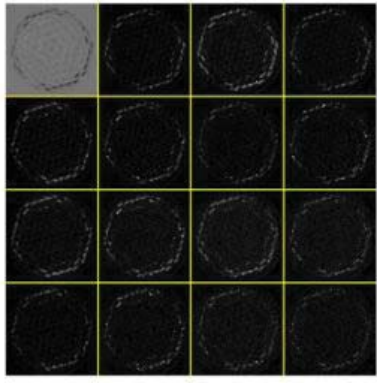

(b)
Figure 3: A defective LED lens image and a defect-free image with appearance faults. a general wavelet transform. It may lead to trouble while using wavelet transform in some implementations which the main message is located in higher frequency coefficients. The frequency resolution of the decomposition filter might not be delicate sufficient to abstract essential message from the decomposed components of the signal. The required frequency resolution could be accomplished by conducting a wavelet packet transform to decompose a signal further. The wavelet packet transform is a generalization of wavelet decomposition that provides an abundant scope of possibilities for signal analysis. Figure 4 demonstrates a normal lens image after being processed 1 and 2 levels of wavelet packet decompositions and the wavelet packet components with their corresponding variable numbers. The partial least squares scheme is applied to discover the elementary connections between two matrices $\mathrm{X}$ and $\mathrm{Y}$, i.e. a latent variableprocedure to build the covarianceformations in the two spaces [18].

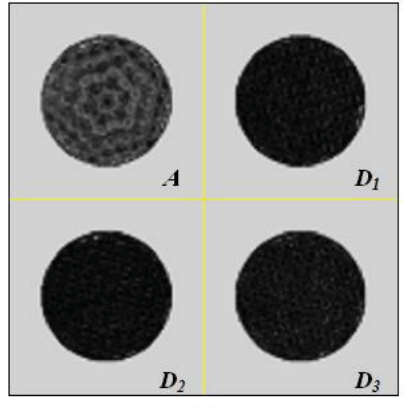

(a)

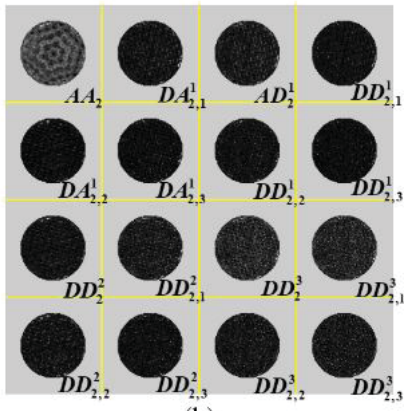

(b)
Figure 4: A normal lens image after being processed (a) 1 level and (b) 2 levels of wavelet packet decompositions and the wavelet packet components with their corresponding variable numbers.

A partial least square methoddiscovers the multi-dimensional direction in the $X$ space that describes the maximum multi-dimensional variance direction in the $Y$ space [15]. The partial least square regression is especiallyadapted when the matrix of predicates has more variables than responses, and when there is existing multi-collinearityin $X$ variables [13]. The partial least squares regression integratescharacteristics from principal component analysis and multiple regression. This forecastis accomplished by abstracting from a group of orthogonal latent variables. In the training process of the partial least squares scheme, we use the intensity values of normal images as the response variable.

After a testing image is transformed to wavelet pack domain, the wavelet characteristics of the sub-band images can be entirely extracted. Then, the suggested partial least squares scheme is used to multivariate transform and data reduction with wavelets features to acquire the latent images. The latent components have much more information than those in the original features. Thus, the latent images are fitted by a regression model to produce a predicted image and then subtract with the original one to obtain the residual image where the visual faults have been segmented. Thence, the visual faults on the un- 
even surfaces of optical lenses can be correctly identified by the suggested method.

This research suggests a wavelet packet transform-based partial least squares method to inspect appearance faults of LED lenses. Five stages are exploited to complete the process of fault detection. Firstly, image preprocessingis executed to remove background region and produce a fused image by integrating the LED lens area with a controlled background to reduce the obstruction of the original one. Secondly, the fused spatial domain image is transformed to the wavelet packet domain and the wavelet packet features of the sub-band images are extracted. Thirdly, the suggested partial least squares scheme is employed to multivariate transform and data reduction with wavelets features to obtain latent images. Fourthly, the latent images are fitted by a regression model to produce a predicted image. Fifthly, the predicted image subtracts with the original image to get the residual image where the intricatefaults have been segmented. Consequently, the visual faults on the unevenappearances of textured LED lenses can be exactly inspected out by the suggested method. Figure 5 shows the procedures of the suggested fault detection method. The dimensional number of the partial least squares is computed by the percentage of variancedescribed and cross validation [14].

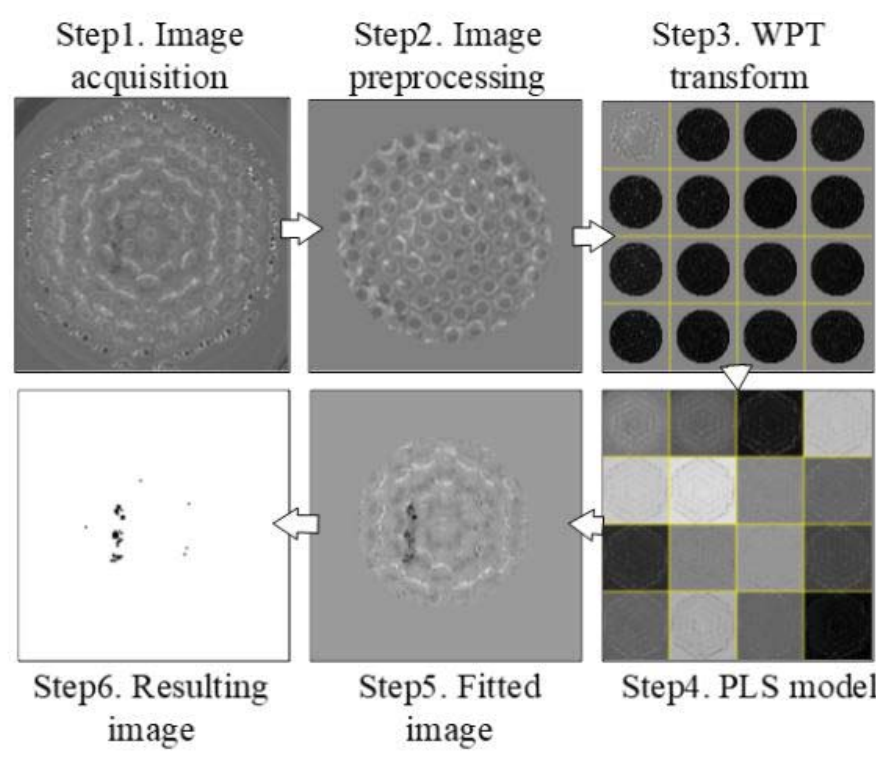

Figure 5: Procedures of the suggested fault detection method.

The cross-validation procedure determines the number of latent variables for obtaining better ability of the model fitness. The index of prediction sum of squares (IPSS) is used to evaluate the model fitness. It is expressed as, where $F(x, y)$ is the input image and is the fitted image. Figure 6 demonstrates a defective LED lens image, the gold standard by censor, and its fitted images, residual images, and resulting images with 1,2, and 3 latent vec-

$$
\operatorname{IPSS}=\sum_{x=0}^{\mathrm{w}} \sum_{y=0}^{\mathrm{h}}[F(x, y)-\hat{F}(x, y)]^{2}
$$

tors. After the latent images are fitted by a regression model to produce a fitted image and then subtract with the original image to get the residual image. The resulting binary images show the detected faults in black by the suggested inspection method. We discover the fitted image with 1 latent vector has a better fault detection of optical lens. The partial least square model with 1 latent vector has the lowest IPSS index. The latent image of the current iteration is obtained from the residual estimate of the previous latent image. The outcomes reveal that the visual faults on uneven surfaces are accurately separated in the resulting image, regardless of optical lenses with textured appearances.

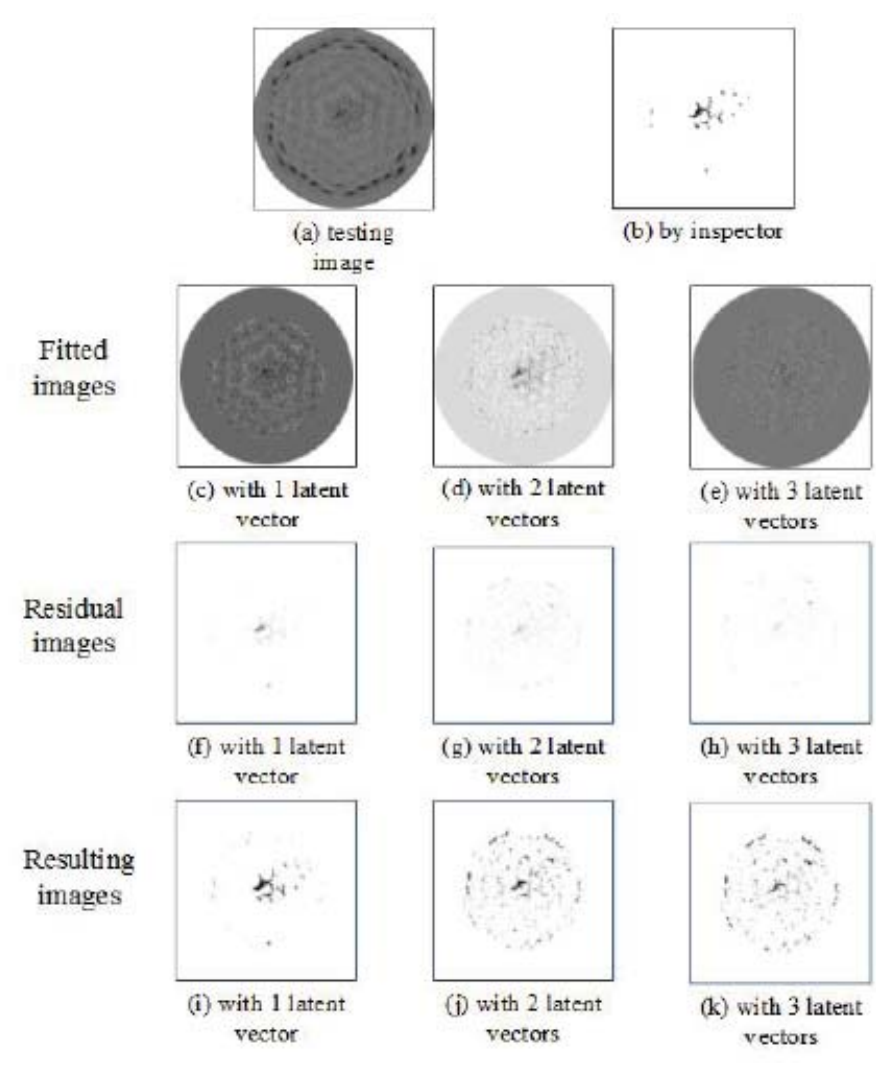

Figure 6: A defective LED lens image, the gold standard by censor, and its fitted images, residual images, and resulting images with 1, 2 and 3 latent vectors

\section{EXPERIMENTS AND DISCUSSION}

To assess the effect of the suggested method, tests were implemented on actual samples of LED lenses supplied by anoptical lens production plant in Taiwan. All trial samples were randomly chosen from the production process of LED lenses. The suggested method is implemented and experiments are conducted to assess its manifestation in inspecting visual faults on uneven surfaces of LED lenses. Examinations are made on 120 LED lens samples comprising 55 normal lenses and 65 defective lenses. Figure 7 shows the image acquisition process (a) testing samples; (b) configuration of experiments; (c) the captured image. Every image of the optical lens has 


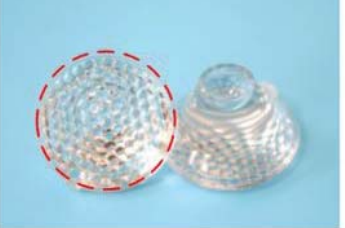

(a)

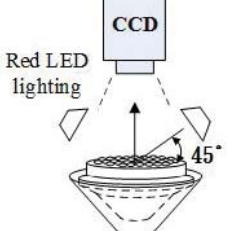

(b)

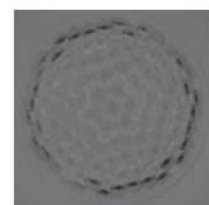

(c)
Figure 7: Image acquisition process (a) testing samples; (b) configuration of experiments; (c) captured image

a size of $512 \times 512$ pixels and an intensity of 8 bits. Algorithms of the suggestedfault detection method are edited in $C$ language and implemented on the 2010version of the $\mathrm{C}++$ Builder compiler on a personal computer (Pentium-4 3.2 GHz and 512 MB DDRII 667Hz-RAM).

To numerically verify the manifestation of the suggested method, we contrast the outcomes of our assessments contrary to those supplied by technical censors (human inspectors). The indices of performance assessment, (1$\alpha)$ and $(1-\beta)$, are utilized to stand forright detection determinations; the larger the two indicators, the more right the detection outcomes [19]. Statistical type I error $\alpha$ describes the probability of generating false warnings, i.e. regarding usualareas as faults. Statistical type II error $\beta$ indicates the probability of generatingmissing warnings, which lose to alert actualfaults. We divide the areas of usualzones detected as faults by the areas of real usualzones to get type I error, and the areas of undetected faults by the areas of realfaults to get type II error. The true classification rate (TCR) is explained as the totals of pixel number of usual textures detected as usual areas and the pixel number of faults detected as defective zones are divided by the total pixel number of a lensarea.

Two known methods commonly used to flaw detection are compared with the suggested method to contrast effects of fault detection. To reveal the fault detection outcomes, Figure 8 displays partial results of inspecting visualfaults by Otsu method [20], Lin and Ho method [7], the suggested method, and the technical censor, separately. The spatial domain technique, the Otsu method, conductsmanywrong judgments (false warnings) on visualfault detection. The frequency domain techniques, the Lin and Ho method, and the suggested method, inspect great majority of the visualfaults and conduct less wrong judgments. Consequently, the frequency domaintechniques excel the spatial domain method in the visualfault detection of opticallenses with textured and uneven surfaces.

Table 1 lists the contrast effects of fault inspection results in the conducted experiments. One spatial domain method and two frequency domain approaches are assessed contrary to the outcomes by technical censors. The average fault detection rates $(1-\beta) \%$ of all testing images by the three methods are, $88.9 \%$ by Otsu method, $50.4 \%$ by Lin and Ho method, and $92.6 \%$ by the suggested method. Nevertheless, the spatial domain method has remarkably higher false warning rates $(\alpha) \%, 10.6 \%$ by Otsu method. Contrarily, the frequency domain techniques have relatively lower false warning rates, $4.23 \%$ by Lin and Ho method, and $0.16 \%$ by suggested method. The suggested method has a highertrue classification rates (TCR)\%, 96.6\%, than those of the Otsu method and Lin and Ho method, $90.5 \%$ and $91.7 \%$, employed to fault detection of LED lens images with uneven surfaces. More specifically, the suggested method not only has a higher fault detection rate but also has a lower false warning rate applied to LED lens images with structural textures.

The average execution time for handling an image with size of 512 x 512 pixelsis asfollows: 0.062 secondsbyOtsu method, 1.458 secondsby Lin and Ho method,and 0.166 secondsby thesuggested method. The average processing time of the suggested method ismore than
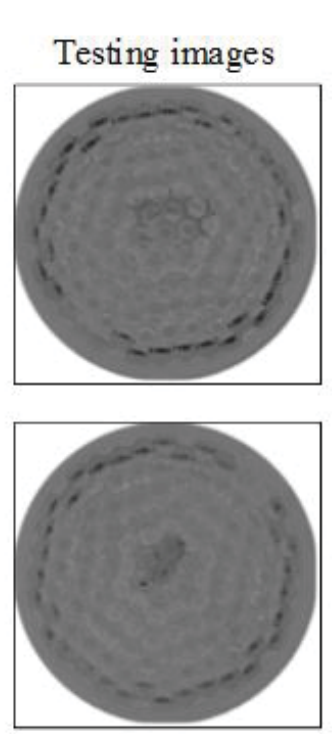
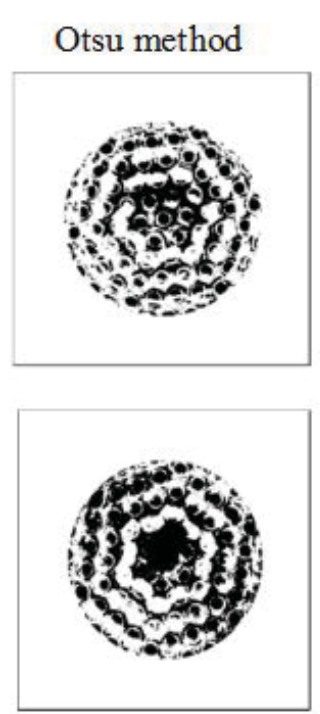
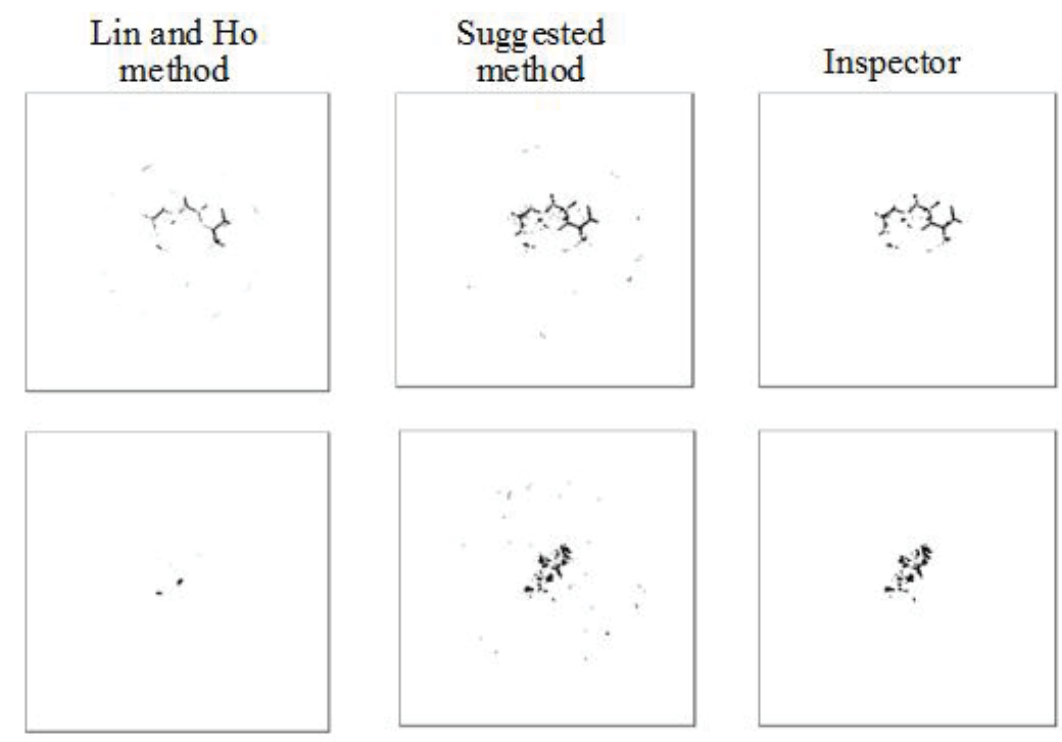

Figure 8: Resulting examples of LED lens fault inspection by Otsu method, Lin and Ho method, the suggested method, and technical censor. 
Table 1: Contrast effects table of three fault inspection methods.

\begin{tabular}{|l|l|l|l|l|l|}
\hline \multicolumn{2}{|l|}{ Inspection methods } & $\begin{array}{l}\text { False warning rate } \\
\alpha(\%)\end{array}$ & $\begin{array}{l}\text { Fault detection } \\
\text { rate } 1-\beta(\%)\end{array}$ & $\begin{array}{l}\text { True } \\
\text { classification rateTCR(\%) }\end{array}$ & $\begin{array}{l}\text { Processing time } \\
(\mathrm{sec})\end{array}$ \\
\hline $\begin{array}{l}\text { Spatial } \\
\text { domain }\end{array}$ & Otsu method & 10.6 & 88.9 & 90.5 & 0.062 \\
\hline $\begin{array}{l}\text { Frequency } \\
\text { domain }\end{array}$ & $\begin{array}{l}\text { Lin and Ho } \\
\text { method }\end{array}$ & 4.23 & 50.4 & 91.7 & 1.458 \\
\cline { 2 - 6 } & $\begin{array}{l}\text { Suggested } \\
\text { method }\end{array}$ & 0.16 & 92.6 & 96.6 & 0.166 \\
\hline
\end{tabular}

eight times shorterthan that ofthe Lin and Ho method. Computational complexity is a measure of resources necessary for implementing an algorithm in computer science. When the essence of the resources is not clearly given, this is usually the time needed for executing the algorithm. The execution time can also be expressed as the number of needed fundamental operations. To analyze the computational complexityof the suggested partial least squares scheme, some major parameters needed to be set including the dimensions of latent variables, the maximum iterations when each dimension is solved, the number of sample pairs in the training set, and the number of total categories. The cross validationof the partial least squares method offers an easy and uncomplicated stopping rule and makes it brief to contrastthe estimates of distinct methods. In summary, the suggested method conquers the problems of inspecting visual faults on optical lens images with textured and uneven surfaces and efficiently overbears in its ability of accurately differentiating visual faults from usual areas.

\section{CONCLUSION}

Computer vision technologies enhance product quality and productivity, and offer competitive merits to industries that utilize these techniques. This study is devoted to the current scientific and technical research. We first review the literature on optical methods of image processing for fault detection on optical elements and confirm the importance and urgency of the development. Then, we introduce a wavelet packet transform-based partial least squares method for the computer-aided inspection of visual faults on textured and uneven surfaces of optical lenses. Actual LED lenses are employed as examined samples and experiments with large sample size are implemented in an actual inspection circumstance to confirm theperformance of the suggested method. The created models of digital image processing allow to justify localization of faults on LED lens surfaces. Thus, the intricate faulted embedded on the textured and uneven surfaces can be correctly identified by the suggested method. Experimental outcomes demonstrate that the suggested method accomplishes a higher probability of accurately differentiating visual faults from usual areas and a lower probability of wrongly inspecting usual areas as faults on surfaces of optical lenses. Further study may expand the suggested approach to inspect visual faults on the surfaces with distinct textural patterns.

\section{ACKNOWLEDGEMENTS}

This study was sponsored by the Ministry of Science and Technology of Taiwan under the grant No. MOST1042221-E-324 -010.

\section{REFERENCES}

1. Lin, H.D.,Chiu, Y.P.,Lin, W.T. (2013). An innovative approach for detection of slight surface variations on capacitor chips.International Journal of Innovative Computing Information and Control, 9(5), 18351850.

2. Chiu, Y.P.,Lo, Y.C.,Lin, H.D. (2017). Hough transform based approach for surface distortion flaw detection on transparent glass.International Journal of Applied Engineering Research, 12(19), 8150-8159.

3. Lin, H.D.,Chiu, Y.P. (2010).RBF network and EPC method applied to automated process regulations for passive components dicing.International Journal of Innovative Computing Information and Control, 6(11), 5077-5091.

4. Lin, H.D.,Hsieh, K.S. (2018). Detection of surface variations on curved mirrors of vehicles using slight deviation control techniques.International Journal of Innovative Computing Information and Control, 14(4), 1407-1421.

5. Chiu, Y.P.,Lin, H.D. (2018).Creation of image models for inspecting visual flaws on capacitive touch screens.Journal of Applied Engineering Science, 16(3), 333-342.

6. Li, W.C.,Tsai, D.M. (2012). Wavelet-based defect detection in solar wafer images with inhomogeneous texture.Pattern Recognition, 45, 742-756.

7. Lin, H.D.,Ho, D.C. (2007). Detection of pinhole blemishes on chips and wafers using DCT enhancement in computer vision system. International Journal of Advanced Manufacturing Technology, 34(5-6), 567-583.

8. Lin, H.D.,Chiu, S.W.(2011). Flaw detection of domed surfaces in LED packages by machine vision system.Expert Systems with Applications, 38(12), 15208-15216. 
9. Chiu, Y.P., Lin, H.D. (2013). An innovative blemish detection system for curved LED lenses.Expert Systems with Applications, 40(2), 471-479.

10. Mallat, S.G. (1989). A theory for multiresolution signal decomposition the wavelet representation.IEEE Transactions on Pattern Analysis and Machine Intelligence, 11(7), 674-693.

11. Amiri, G.G.,Asadi, A. (2009). Comparison of different methods of wavelet and wavelet packet transform in processing ground motion records.International Journal of Civil Engineering, 7(4), 248-257.

12. Kim, S.C.,Kang, T.J. (2007). Texture classification and segmentation using wavelet packet frame and Gaussian mixture model.Pattern Recognition, 40, 1207-1221.

13. Wold, H. (1966). Estimation of principal components and related models by iterative least squares. In: Krishnaiah, P. R., Ed., Multivariate Analysis II. Academic Press: New York, 391-420.

14. Geladi, P., Kowalski, B.R. (1986). Partial least squares regression: a tutorial.Analytica Chimica Acta, 185, 1-17.
15. Martens, H.,Naes, T. (1992).Multivariate Calibration, 2nd. Edn, Wiley's publisher, Chichester, 116-165.

16. Li, J., Rao, X., Ying, Y. (2011). Inspection and grading of surface defects of fruits by computer vision. Advanced Materials Research, 317-319, 956-961.

17. Lin, H.D.,Chen, H.L. (2018). Computer-aided industrial inspection of optical components using computer vision technologies. Proceedings of the 2018 International Conference on Image Processing, Computer Vision, \& Pattern Recognition (IPCV 2018), p. 41-44.

18. Helland, I.S. (1990). Partial least squares regression and statistical models.Scandinavian Journal of Statistic, 17, 97-114.

19. Montgomery, D.C.,Runger, G.C. (2007).Applied Statistics and Probability for Engineers. 4th Edition, John Wiley \& Sons, New Jersey, USA.

20. Otsu, N. (1979). A threshold selection method from gray level histogram.IEEE Transactions on Systems, Man and Cybernetics, 9, 62-66. 